\title{
Nano-lignin filled natural rubber composites: Preparation and characterization
}

\author{
C. Jiang, H. He*, H. Jiang, L. Ma, D. M. Jia \\ Department of Polymer Materials and Engineering, South China University of Technology, 510640 Guangzhou, China
}

Received 24 December 2012; accepted in revised form 10 February 2013

\begin{abstract}
This paper presents a novel strategy to prepare nano-lignin and its composites with natural rubber. The nanolignin was ontained by fabricating colloidal lignin-Poly (diallyldimethylammonium chloride) (PDADMAC) complexes (LPCs) via self-assembly technology. The characteristics of LPCs were investigated by zeta potential, dynamic light scattering (DLS), transmission electron microscopy (TEM), Fourier transform infrared spectroscopy (FTIR) and ultraviolet visible (UV-vis) absorption measurements. The results indicated that PDADMAC intensively interacted with lignin by cation- $\pi$ and $\pi-\pi$ interactions, and lignin particles were stable in aqueous solution with an average particle size less than $100 \mathrm{~nm}$. LPCs accelerated the vulcanization of NR/LPCs nanocomposites. Morphological studies and Dynamic mechanical analysis (DMA) showed the homogeneous dispersion of LPCs in the NR matrix and the strong interfacial adhesion between them. The nanoscale dispersion of LPCs significantly enhanced the thermal stability and mechanical properties of NR/LPCs nanocomposites.
\end{abstract}

Keywords: nanocomposites, natural rubber, nano-lignin, cationic polyelectrolyte, self-assembly

\section{Introduction}

Lignin, the second most abundant renewable natural resource next to cellulose, is a highly-branched, three dimensional biopolymer. It consists of three phenylpropanoid units, such as $p$-hydroxyphenyl $(\mathrm{H})$, guaiacyl $(\mathrm{G})$ and syringyl (S) (Figure 1), which are attached to one another by a series of characteristic linkages $(\beta-\mathrm{O}-4, \beta-5, \beta-\beta$, etc.) [1]. The major chemical functional groups in lignin include hydroxyl, methoxyl, carbonyl and carboxyl groups in various amounts and proportions, depending on genetic origin and applied extraction processes [2]. The distinct network structure as well as the presence of the various chemical substituents confers special functional properties to lignin, such as stabilizing effect [3], reinforcing effect, UV-absorption, biodegradability, anti-fungal and antibiotic activity [4-6]. However, the potential of lignin is not clearly valued, because it predominantly obtained as byproducts in pulp production is mainly used as fuel. Fortunately, incorporating into polymeric materials will be a value-added application for lignin [7-10]. Especially in rubber-based materials, lignin is less dense, non-conducting, and being lighter in color compared to carbon black. It appears more amenable for the preparation of light-colored rubber compounds. So, for the past decades great amounts of researches [11-13] and patents [14-18] of lignin as filler added to rubber compounds have investigated carbon black replacement in order to achieve similar reinforcement of rubber composites. Usually, two strategies (dry-milling and co-precipitation) are mostly used for the preparation of lignin-filled rubber composites. Lignin as dry powder straightforward milled into rubber shows almost no reinforcing effect. This is believed to be a result of the

\footnotetext{
${ }^{*}$ Corresponding author, e-mail: pshuihe@scut.edu.cn

(C) BME-PT
} 


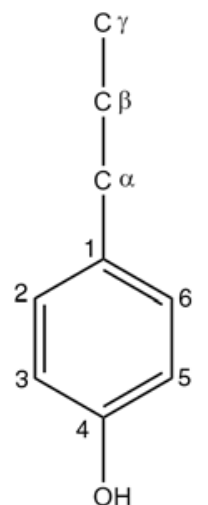

$\mathrm{H}$

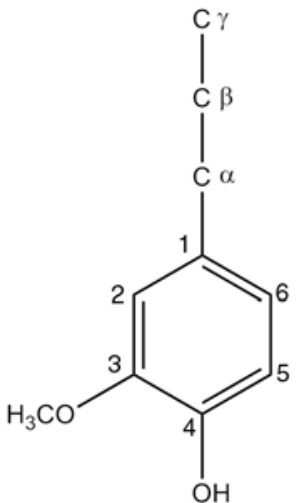

G<smiles>COc1cc(CCCl)cc(OC)c1O</smiles>

S

Figure 1. Three phenylpropanoid units of lignin

lignin particles adhering together by intermolecular hydrogen bonding and thus not being dispersed into the rubber by milling [19]. It is a unique property that lignin is soluble in aqueous alkali because of the ionization of the phenolic hydroxyl and carboxylic groups, such solutions being compatible with rubber latex in all proportions. Furthermore, the co-precipitation of the lignin and the rubber from the mixing solution with acids is possible in the same $\mathrm{pH}$ range [20]. In comparison to dry-milling method, the distribution of the lignin in the rubber compounds prepared by co-precipitation method is more homogeneous and the particle size is finer. Even so, the lignin is still unable to be dispersed at nanoscale. Nevertheless, the reinforcing effect of lignin for rubber compounds intensively depends on particle size and strong interfacial bonding with rubber matrix. In most reports, the lignin shows little reinforcing effect on nonpolar rubber matrix and even deteriorates the performance of rubber composites [21, 22]. To the best of our knowledge, there is no report on nanoscale lignin reinforced natural rubber. Therefore, we highlight the fabrication of nano-lignin and the nanoscale distribution of lignin in rubber matrix by a novel strategy in this paper. Lignin can be viewed as an anionic polyelectrolyte when phenolic hydroxyls and carboxylic groups are ionized. Usually, three types of polyelectrolyte complexes can form: soluble, colloidal and coacervate [23]. In previous publications, the studies on the formation of polyelectrolyte complexes between cationic polyelectrolytes and anionic lignin aim to improve wastewater-treatment efficiency [24, 25], increase the efficiency of retention aids and enhance the strength of papers in papermaking [26]. As a consequence, the lignin-cationic polyelectrolyte com- plexes are commonly coacervates for those purposes. However, the complexes must be colloidal to mix with natural rubber latex (NRL) and produce $\mathrm{NR} /$ lignin nanocomposites. In the present work, the formation and characteristics of colloidal LPCs were investigated and the adsorption mechanism of PDADMAC onto the lignin surface was discussed. The morphology of the NR/lignin composites was also observed by SEM. The mechanical performance and vulcanization behaviors were studied, as well as the thermal and thermo-oxidative stability.

\section{Experimental section}

\subsection{Materials}

Lignin was industrial sulfate lignin (micron scale), purchased from Shandong Tralin Pape Co., Ltd., China. The average molecular weight of sulfate lignin was 3801 with the polydispersity index of 2.15. PDADMAC (100 000-200 000; $20 \mathrm{wt} \%$ in water) was obtained from Sigma-Aldrich (SigmaAldrich, Louis, MO). NRL stabilized by ammonia with a total solid content of $64.5 \%$ was provided by the Tropical Crops Research Center of Zhanjiang, China. Sodium hydroxide, hydrochloric acid and sulfuric acid were analytical grade, purchased from Guangzhou Chemical Reagent Factory, China. The reagents used in rubber formula such as sulphur, stearic acid, zinc oxide, N-tert-butyl-2-benzothiazole sulfonamide (CBS), were industrial grade, kindly provided by South China Rubber Tyre co., Ltd.

\subsection{Purification of the industrial sulfate lignin}

The industrial sulfate lignin was dissolved in a beaker with deionized water followed by adding $\mathrm{NaOH}$ to adjust the $\mathrm{pH}$ to approximately 13 . The 
resulting solution was centrifuged to exclude fibril or insoluble impurities before slowly titrating with $1.0 \mathrm{~N}$ sulfuric acid to a final $\mathrm{pH}$ of approximately 2 with mechanical stirring. Subsequently, the slurry was placed in water bath at $70^{\circ} \mathrm{C}$ for $2 \mathrm{~h}$ to accelerate lignin agglomeration then filtered. The lignin was washed with deionized water three times and dried in a vacuum oven at $50^{\circ} \mathrm{C}$ for $24 \mathrm{~h}$ followed by extraction with pentane to remove the organic impurities. Finally, the purified lignin was dried at $50^{\circ}$ Cand stored under reduced pressure.

\subsection{Preparation of sample solution}

Purified sulfate lignin was dissolved in deionized water at a mass concentration of $0.5 \%$ and the $\mathrm{pH}$ was adjusted to 12. PDADMAC aqueous solution was diluted to $2 \mathrm{wt} \%$ with deionized water followed by adjusting the $\mathrm{pH}$ to 12 . The various mass of sulfate lignin solution was dropped slowly into the PDADMAC solution with vigorous stirring, leading to formation of colloidal LPCs with different mass ratios of lignin to PDADMAC.

\subsection{Preparation of $\mathrm{NR} /$ /ignin compounds}

The NRL was diluted to a solid content of $10 \%$ and its $\mathrm{pH}$ was adjusted to 12 with $1 \mathrm{~N} \mathrm{NaOH}$. After that, a desired amount of the lignin solution or the colloidal LPCs solution was then dropped into NRL under vigorous mechanical stirring. The resulting solution was co-precipitated by adjusting the $\mathrm{pH}$ to 2 with $1 \mathrm{~N}$ sulfuric acid. Finally, the obtained mixtures were filtered, water washed and dried in a vacuum oven at $50^{\circ} \mathrm{C}$.

All the rubber compositions were summarized in Table 1. The dried composites and other additives were mixed on an open two-roll mill. Then the compounds were vulcanized in a standard mold at $143 \pm 1{ }^{\circ} \mathrm{C}$ for optimum vulcanization time, which was determined by the U-CAN UR-2030 vulcameter (Taipei, Taiwan).

\subsection{Characterizations}

The particle size of lignin and colloidal LPCs as a function of solution $\mathrm{pH}$ was determined by DLS using a N5 Submicron Particle Size Analyzer (Beckman Coulter) with a detection angle of $90^{\circ}$ at $25^{\circ} \mathrm{C}$. The effect of mass ratio of lignin to PDADMAC on the particle size of LPCs was also studied by DLS. All samples were repeatedly measured three times. The zeta potential measurements were carried out on Malvern Zetasizer Nano ZS90.

The UV-vis absorption measurements were performed with a UV-vis spectrophotometer (UV756CRT, Shanghai Youke Instrument CO. Ltd., China). Deionized water was scanned at the same wavelength as a baseline.

The sample solutions of lignin or LPCs were diluted with $0.01 \mathrm{~N} \mathrm{NaOH}$ to a certain mass concentration of $0.05 \%$ (based on lignin) for DLS and zeta potential measurements or $0.01 \%$ for UV-vis absorption measurements. A few drops of either $0.1 \mathrm{~N}, 0.01 \mathrm{~N}$ or $0.001 \mathrm{~N} \mathrm{HCl}$ was added to the diluted sample solutions to adjust the $\mathrm{pH}$ before the measurements. The samples for TEM measurement were prepared by dropping sample solution on $\mathrm{Cu}$ grids of 200 mesh and then observed on a Hitachi H-7650 instrument (Hitachi, Japan) with an accelerating voltage of $80 \mathrm{kV}$.

FTIR was recorded in transmission mode with a Bruker Vertex 70 FTIR spectrometer at a spectral resolution of $4 \mathrm{~cm}^{-1}$ and 32 scans. Then $\mathrm{KBr}$ pellets of lignin, PDADMAC and LPCs were prepared (200 mg KBr : 1-2 mg sample) with pressure.

Scanning electron micrographs (SEM) of the composites were taken with a Nova NanoSEM 430 instrument (FEI, Netherlands) at an acceleration voltage of $10 \mathrm{kV}$. The fracture surface was obtained by splitting bulk sample being quenched in liquid nitrogen. Before the observation, a thin gold was evaporated on the fractured surface.

Table 1. Recipe of NR/lignin compounds ${ }^{\mathrm{a}}$

\begin{tabular}{|l|c|c|c|c|c|c|c|c|c|}
\hline \multirow{2}{*}{$\begin{array}{c}\text { Ingredients } \\
\text { [phr] }\end{array}$} & \multicolumn{10}{|c|}{ Sample code } \\
\cline { 2 - 11 }$y$ & L-0 & L-1 & L-3 & L-5 & L-7 & LPCs-1 & LPCs-3 & LPCs-5 & LPCs-7 \\
\hline NR & 100 & 100 & 100 & 100 & 100 & 100 & 100 & 100 & 100 \\
\hline Lignin & 0 & 1 & 3 & 5 & 7 & 0 & 0 & 0 & 0 \\
\hline LPCs $^{c}$ & 0 & 0 & 0 & 0 & 0 & 1 & 3 & 5 & 7 \\
\hline
\end{tabular}

arubber ingredients: $\mathrm{ZnO} 5$, Stearic acid 2, CBS 2, S 2.

${ }^{\mathrm{b}} \mathrm{phr}$, parts per hundred of rubber by weight.

cthe ratio of lignin to PDADMAC is 0.45 , and the amount of LPCs in the formula refers to the weight of lignin in the LPCs. 
The curing characteristics of the compounds were determined at $143^{\circ} \mathrm{C}$ by U-CAN UR-2030 vulcameter. After the compounds were vulcanized for vulcanization time $\left(T_{\mathrm{c} 90}\right)$ at $143 \pm 1^{\circ} \mathrm{C}$, tensile and tear tests of the vulcanizates were performed according to ISO 37-2005 and ISO 34-2004, respectively. The crosslink density of the vulcanizates was measured according to the method described by Gregorová et al. [27].

DMA spectra of the samples were obtained by using a DMA 242D dynamic mechanical analyzer (NETZSCH Company, Germany). The specimens with the size of $30 \mathrm{~mm} \times 6 \mathrm{~mm} \times 4 \mathrm{~mm}$ were analyzed in tensile mode at a constant frequency of $1 \mathrm{~Hz}$, a strain of $0.5 \%$, and a temperature range from -130 to $50^{\circ} \mathrm{C}$ at a heating rate of $3^{\circ} \mathrm{C} / \mathrm{min}$.

A Perkin-Elmer Pyris 1 TGA thermogravimetric analyser (TGA) (Perkin-Elmer, Fremont, USA) was used for thermal and thermo-oxidative stability measurement. In nitrogen, the measurement was carried out from room temperature to $700^{\circ} \mathrm{C}$ at a heating rate of $10^{\circ} \mathrm{C} / \mathrm{min}$. In air, the TGA analysis was carried out at the same temperature range and heating rate. The flow rate of the carrying was $20 \mathrm{~mL} / \mathrm{min}$.

\section{Results and discussion}

\subsection{Effect of $\mathrm{pH}$ on the particle size of lignin}

The aggregation and hydrodynamic radius of colloidal lignin in aqueous solution can be influenced by many factors, such as $\mathrm{pH}[28,29]$, temperature [30], electrolyte [31,32], concentration of lignin [33] and surfactant [34]. Due to the ionizable phenolic hydroxyls and carboxylic groups in lignin macromolecules, the association and particle size of lignin are very sensitive to the solution $\mathrm{pH}$. Figure 2 shows the effect of the solution $\mathrm{pH}$ on the particle

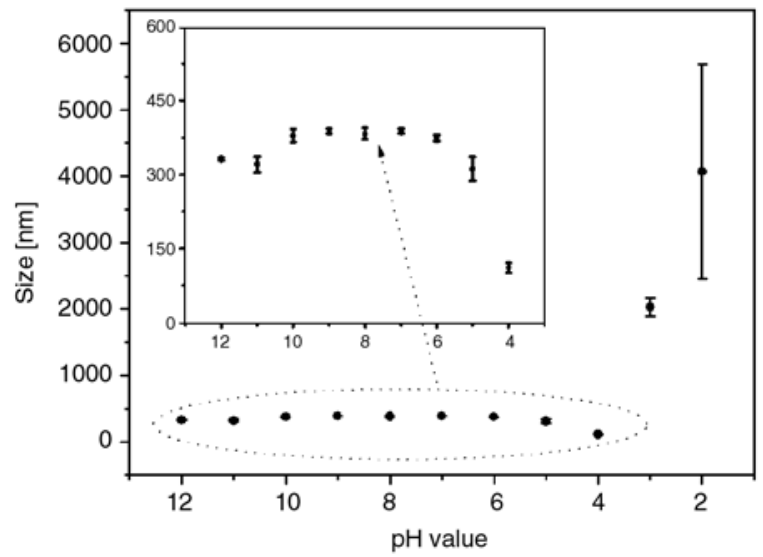

Figure 2. The effect of the solution $\mathrm{pH}$ on the particle size of lignin size of lignin. As the $\mathrm{pK}_{\mathrm{a}}$ of the phenolic hydroxyls on lignin is higher than 10.2 [35], they are not ionized when the $\mathrm{pH}$ is below 10. But lignin still has negative charges at alkaline and neutral $\mathrm{pH}$ that can be attributed to the ionized carboxylic groups. Therefore, the lignin is still stable at that $\mathrm{pH}$ range from 10 to 7. At acidic $\mathrm{pH}$, the degree of dissociation of carboxylic groups decreases with decreasing $\mathrm{pH}$. Consequently, lignin macromolecules shrink at the pH range from 6 to 4 (i.e., from about 380 to about $110 \mathrm{~nm}$ ). When the $\mathrm{pH}$ is low enough and carboxylic groups are completely protonated, the serious aggregation of lignin particles is unavoidable. It can be attributed that the attractive forces including hydrophobic interactions, hydrogen bonding interactions [28] and $\pi-\pi$ interactions [36-39] surpass the electrostatic repulsive forces. Conceivably, the lignin particles will aggregate and form agglomerates in rubber matrix, when the NRL and lignin is co-precipitated by adding acid.

\subsection{Characteristics of colloidal LPCs}

It is generally known that oppositely charged polyelectrolytes form complexes over a broad range of stoichiometric ratios and that the complexes tend to be water-soluble unless they are nearly stoichiometric [23]. In this work, the colloidal LPCs were fabricated by dropwise adding the alkali solution of lignin into the PDADMAC solution. Hence, the formed LPCs were positively charged and water-soluble until the mass ratio of lignin to PDADMAC exceeded stoichiometric point. However, the NRL particles were natively charged at the $\mathrm{pH}$ of 12 , as the protein molecules absorbed on the surface of NRL particles contained carboxylic and amino groups and the carboxylic groups would be ionized at that $\mathrm{pH}$. When the LPCs solution were added into NRL, the natively charged NRL were subsequently adsorbed onto the positively charged LPCs via electrostatic self-assembly, which would suppress the aggregation of lignin and finally resulted in homogeneous distribution of lignin in NR matrix (see Figure 3). However, excess PDADMAC adsorbed onto lignin is unnecessary. If present in excess, it will prematurely flocculate NRL and reduce the loading of lignin incorporated into rubber matrix. To optimize the fabrication of LPCs, the effect of mass ratio of lignin to PDADMAC on particle size of LPCs was investigated (Figure 4). The particle size of LPCs is consistently reduced (from about 400 to about $180 \mathrm{~nm}$ ) with the increas- 


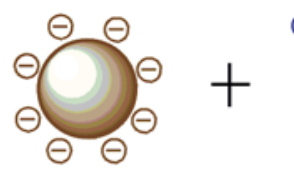

Lignin

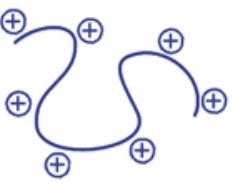

PDADMAC

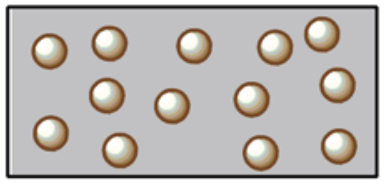

NR/lignin nanocomposites
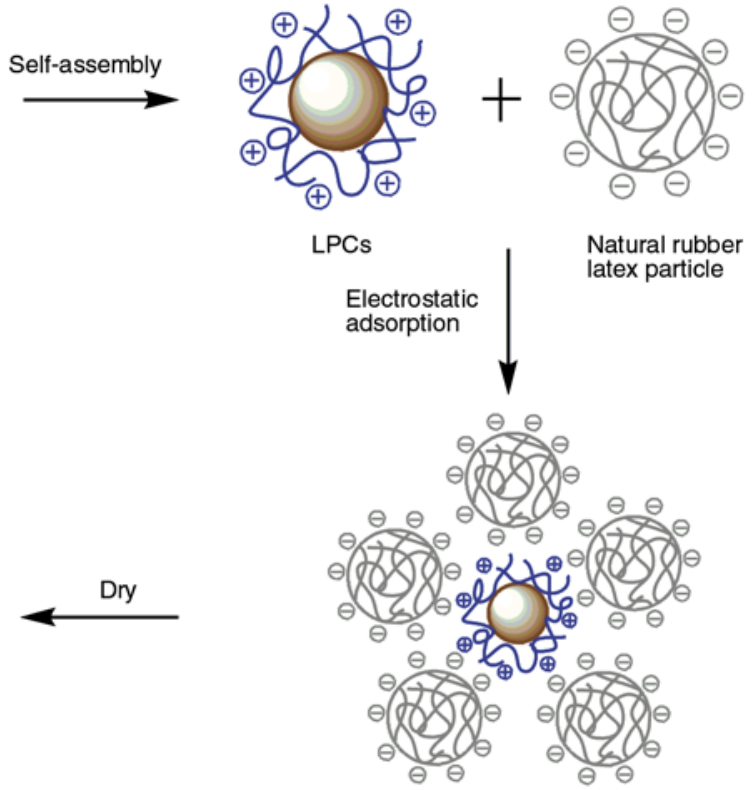

Figure 3. Schematic illustration of process for NR/Lignin nanocomposites

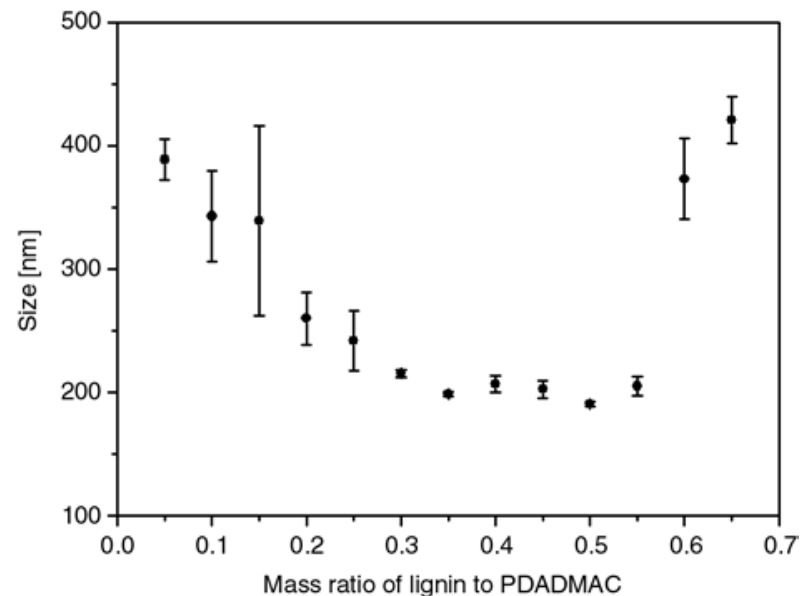

Figure 4. The effect of mass ratio of lignin to PDADMAC on particle size of LPCs at $\mathrm{pH}$ of 12

ing lignin/PDADMAC ratio (from 0.05 to 0.5 ). It should be noted that the minimum particle size of LPCs is still larger than that of the collapsed lignin at the $\mathrm{pH} 4$ (about $110 \mathrm{~nm}$ ). The particle size should be the size of collapsed lignin plus the thickness of absorption layer of PDADMAC onto lignin surface. A significantly increasing particle size follows when lignin and positively charged LPCs are present. Similarly, the particle size of colloidal LPCs as a function of solution $\mathrm{pH}$ was investigated (Figure 5). It can be seen that the particle size of colloidal LPCs with lignin/PDADMAC ratios of 0.45 and 0.5 is very close and stable at the $\mathrm{pH}$ range from 12 to 2 . It is quite different from the results in Figure 2. Hence, the lignin can be dispersed at nanoscale in NR matrix when acid is used to co-precipitate rub-

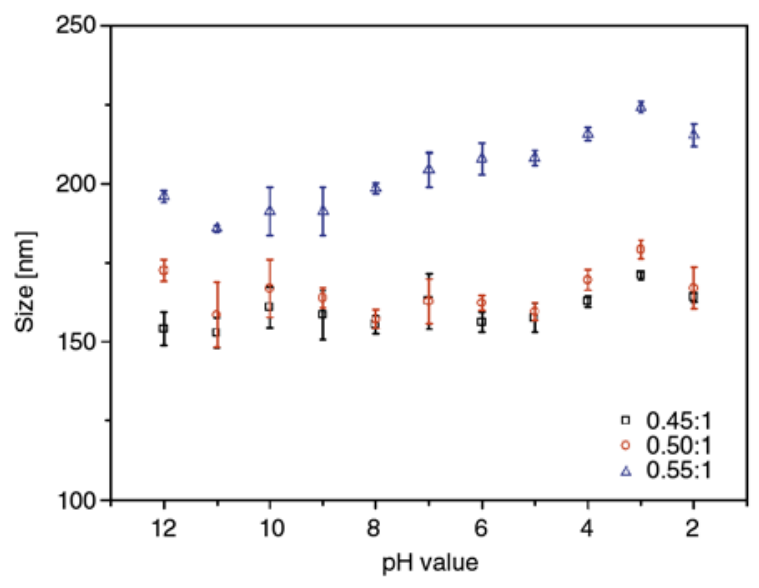

Figure 5. The particle size of colloidal LPCs with different mass ratios of lignin to PDADMAC as a function of solution $\mathrm{pH}$

ber latex and LPCs. In the case of colloidal LPCs with a lignin/PDADMAC ratio of 0.55 , a slightly increasing particle size with decreasing $\mathrm{pH}$ indicates the association of LPCs. Furthermore, the original particle size of colloidal LPCs with a lignin/ PDADMAC ratio of 0.55 is larger than that of two others, indicating the partial association of LPCs at the beginning of formation of colloidal LPCs.

In fact, the results from DLS measurements are apparent hydrodynamic diameters, obtained from the measured diffusion coefficients using the StokesEinstein relationship. The particle size form DLS is always larger than actual size [40], especially in colloidal LPCs system. Adsorbed PDADMAC will significantly increase the hydrodynamic diameter of 


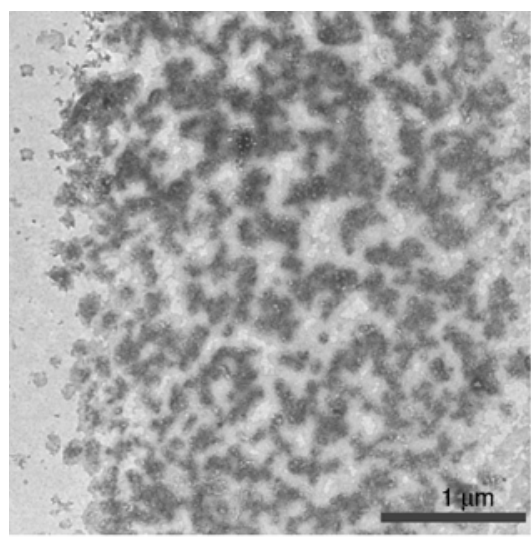

a)

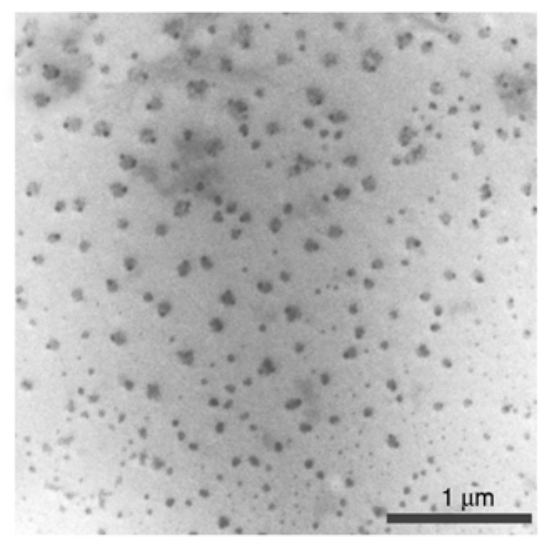

b)

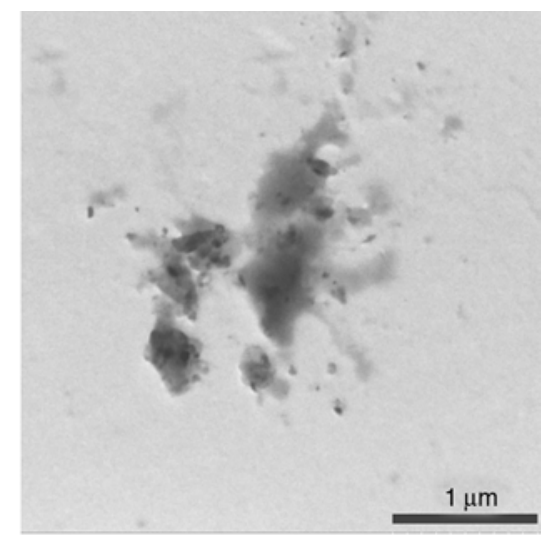

c)

Figure 6. TEM photos of lignin (a), LPCs with a lignin/PDADMAC ratio of 0.5 (b) and LPCs with a lignin/PDADMAC ratio of 0.6 (c)

lignin because of the length of PDADMAC molecular chain. In a polydisperse system, results from DLS measurements give more or less the diameters of the largest aggregates, and not a weight average or size average [34]. To assess the actual size of lignin and LPCs, their TEM photos are shown in Figure 6. It can be seen that serious aggregation and amorphous profile are present in the sample of lignin (Figure 6a). Relatively, LPCs with a mass ratio of 0.5 are stable and individually dispersed with an average size $60.8 \mathrm{~nm}$, which was calculated by Nano Measurer (Figure 6b). However, LPCs become larger as the lignin/PDADMAC ratio rises to 0.6 (Figure 6c), which is similar to the results from DLS. It should be attributed to the bridging flocculation of lignin and PDADMAC. Hence, the lignin/PDADMAC ratio should be less than 0.55 to fabricate nanoscale and stable lignin.

The zeta potential of colloidal LPCs was measured in this work in order to study the charge state of colloidal LPCs surface (Figure 7). The zeta potential of lignin in aqueous alkali had been measured $(-22.3 \mathrm{mV} ; \mathrm{pH}=12)$. Due to the adsorption of positively charged PDADMAC onto lignin surface, there were excess positive charges on the surface of colloidal LPCs. With increasing the lignin/PDADMAC ratio, the zeta potential of colloidal LPCs monotonically decreases, which indicates that charge density on the LPCs surface gradually decreases with increasing lignin/PDADMAC ratio. However, the charge on LPCs surface is still positive, when the lignin/ PDADMAC ratio is less than 0.55 . Therefore, positively charged LPCs can adsorb negatively charged natural rubber latex in latex compounding proce-

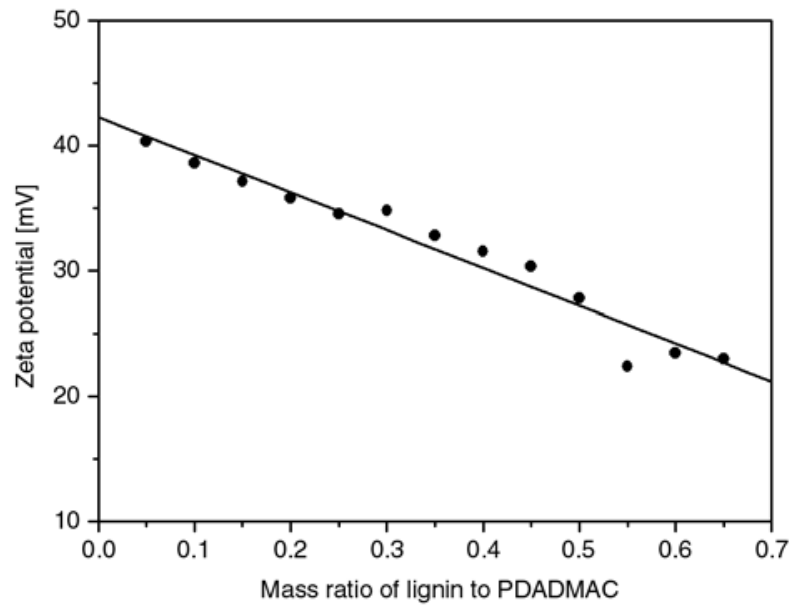

Figure 7. The zeta potential of LPCs as a function of mass ratio of lignin to PDMADMAC at $\mathrm{pH}$ of 12

dure, which will further suppress the association of lignin.

The UV-vis absorption spectra of colloidal LPCs as a function of solution $\mathrm{pH}$ offer a powerful proof to further clarify the adsorption mechanism of PDADMAC to lignin. As shown in Figure 8a, the absorption bands located at 240 and $288 \mathrm{~nm}$ are assigned to $\mathrm{K}$ and $\mathrm{B}$ bands of $\pi-\pi^{*}$ transitions of the aromatic rings in lignin linked by ionized phenolic hydroxyls and carboxylic groups. Obviously, the K band disappears and the $\mathrm{B}$ band shifts to $276 \mathrm{~nm}$ with decreased absorption intensity as the solution $\mathrm{pH}$ changes from 12 to 7, which is the result of protonation of phenolic hydroxyls. Herein, the UV-vis spectrum of lignin at acidic $\mathrm{pH}$ is not shown due to the solubility of lignin. For LPCs, the similar tendency is found in Figure $8 \mathrm{~b}$. Considering the dissociation constant of phenolic hydroxyls, the decreased intensity from 

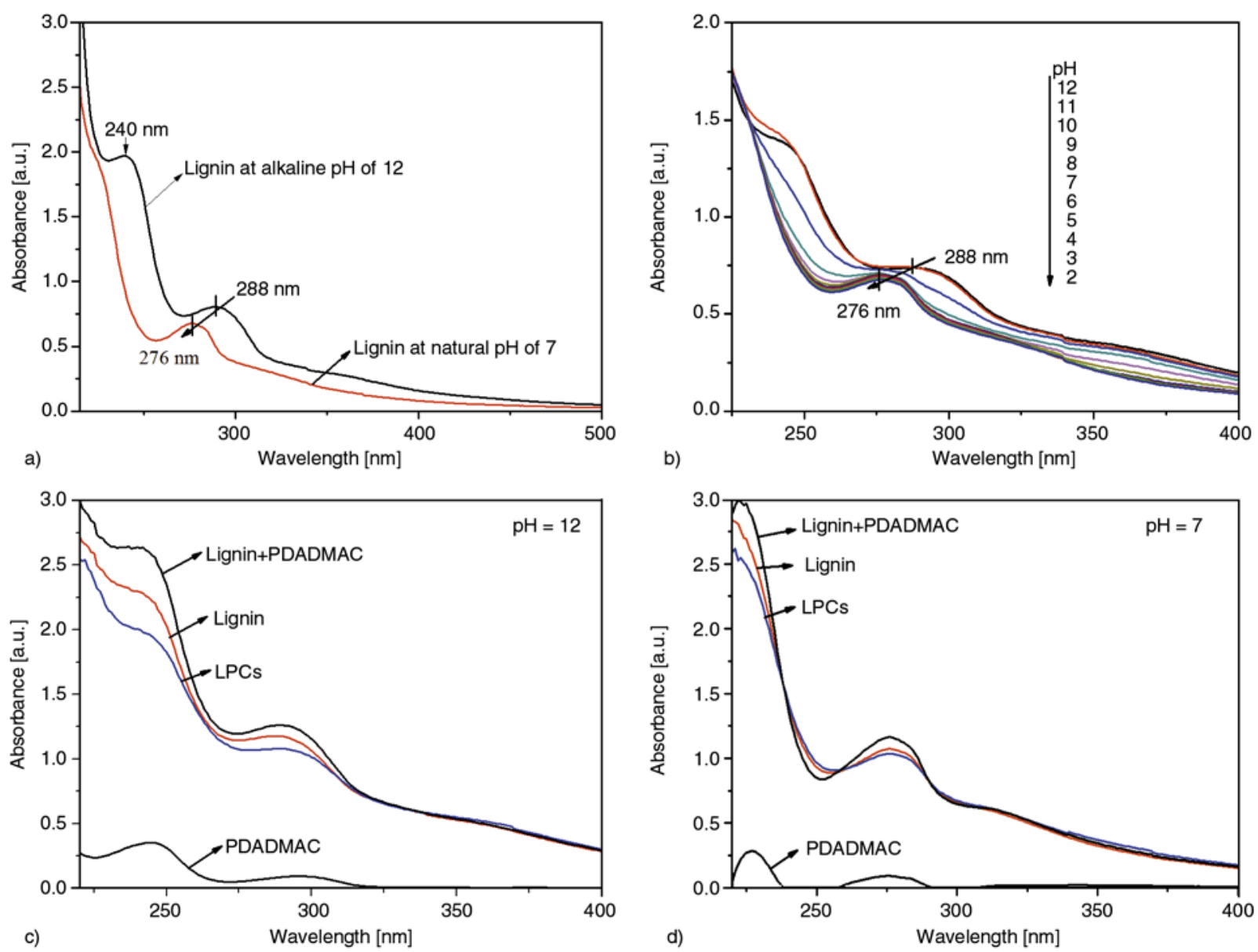

Figure 8. (a) The UV-vis absorption spectra of lignin as functions of solution pH; (b) the UV-vis absorption spectra of LPCs as functions of solution $\mathrm{pH}$; (c) the UV-vis absorption spectra of LPCs $(0.05 \mathrm{~g} / \mathrm{L}$ lignin, $0.11 \mathrm{~g} / \mathrm{L}$ PDADMAC), lignin $(0.05 \mathrm{~g} / \mathrm{L})$ and PDADMAC $(0.11 \mathrm{~g} / \mathrm{L})$ at $\mathrm{pH}$ of 12 ; (d) the UV-vis absorption spectra of LPCs $(0.05 \mathrm{~g} / \mathrm{L}$ lignin, $0.11 \mathrm{~g} / \mathrm{L}$ PDADMAC), lignin $(0.05 \mathrm{~g} / \mathrm{L})$ and PDADMAC $(0.11 \mathrm{~g} / \mathrm{L})$ at $\mathrm{pH}$ of 7 . Note that the curves of lignin + PDADMAC are the superposed spectra of lignin and PDADMAC.

$\mathrm{pH} 10$ to 7 may be ascribed to a hysteresis of protonation of phenolic hydroxyls. Unlike phenolic hydroxyls, carboxylic groups have less influence on the ultraviolet light absorption of lignin. So, no decreased intensity was observed at acidic $\mathrm{pH}$. However, the protonation of carboxylic groups is possible at acidic $\mathrm{pH}$ since phenolic hydroxyls can be protonized. The hypochromic effect of colloidal LPCs with decreasing $\mathrm{pH}$ indicates that $\mathrm{H}$ ions can penetrate through the adsorption layer of PDADMAC and combine with ionized functional groups. In other words, the ionized functional groups do not form short-distance ion pairs with PDADMAC. This result is coincided with others' work [24]. However, the colloidal LPCs could be stabilized by PDADMAC at acidic $\mathrm{pH}$, indicating other interactions between lignin and PDADMAC except ion-ion interactions. Figure $8 \mathrm{c}$ and $8 \mathrm{~d}$ show the UV-vis spectra of LPCs, as well as the spectra of its corresponding components, such as lignin and PDADMAC, respectively at
$\mathrm{pH}$ of 12 and 7. The superposed spectra of lignin and PDADMAC are also shown for comparison with LPCs. The obvious differences in the maximum absorption and the shape between the spectra of LPCs and the superposed spectra indicate the strong interactions between lignin and PDADMAC. It should be attributed to cation- $\pi$ interactions, widely reported between benzene rings and polycations $[41,42]$, which can result in charge-transfer from the aromatic rings in lignin to PDADMAC.

For the driving forces of adsorption of PDADMAC onto lignin, Pillai and Renneckar [43] considered that cation- $\pi$ interactions as driving forces contribute to the adsorption besides ion-ion interactions. In this work, another noncovalent interaction, $\pi-\pi$ interaction between an unsaturated contaminant in the PDADMAC chain (Figure 9a) and lignin, was revealed by FTIR (Figure 9b). The peaks at 1636 and $1474 \mathrm{~cm}^{-1}$ in PDADMAC, corresponding to the stretching of the double bonds in the contaminant, 


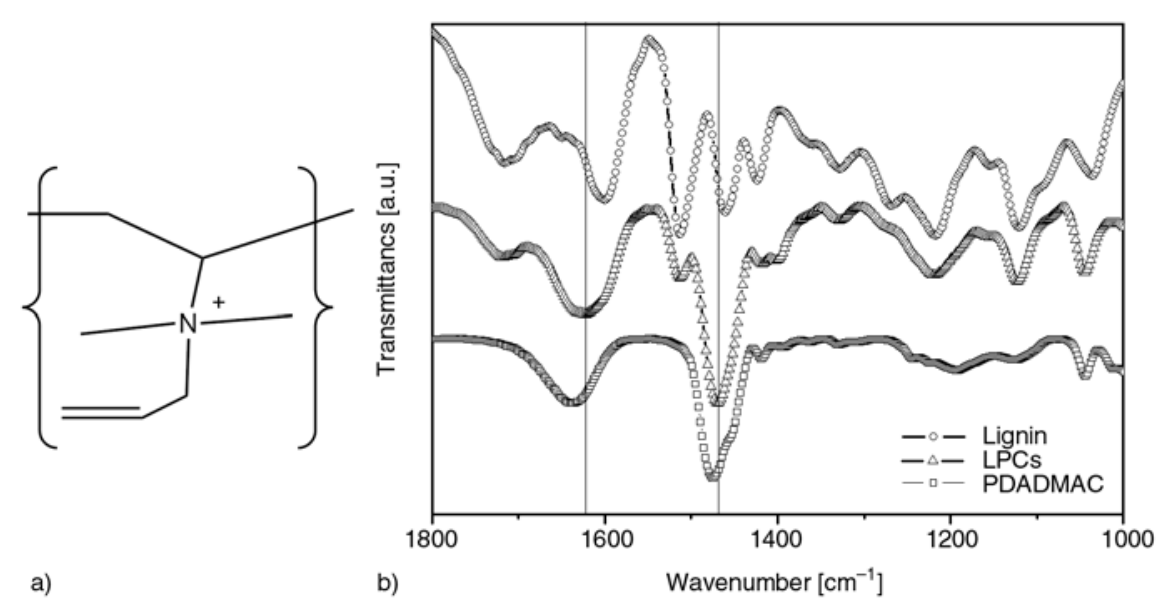

Figure 9. Chemical structure of the contaminate in PDADMAC (a) and FTIR spectra of lignin, LPCs and PDADMAC (b)

respectively shift to 1623 and $1470 \mathrm{~cm}^{-1}$ in LPCs, which are also different from the peaks at 1600 and $1460 \mathrm{~cm}^{-1}$ in lignin. The shifts indicate that the double bonds both in the contaminant and lignin are in a different environment. Because of a great amount of aromatic units in lignin interacting with each other via $\pi-\pi$ interactions, similarly, the double bound in the contaminant can also stack with the aromatic rings in lignin. The similar result was also observed by Yang et al. [44] in the system of PDADMAC/Multiwalled Carbon Nanotubes.

The additional driving forces, $\pi-\pi$ interactions, enhance the adhesion of PDADMAC on lignin surface. Furthermore, the molecule chain skeleton of PDADMAC is similar to that of NR, which suggests a good compatibility between PDADMAC and NR. Therefore, the interfacial bonding between lignin and NR matrix in NR/LPCs composites is better than that in NR/lignin composites.

\subsection{Morphology of NR/lignin and NR/LPCs composites}

Lignin dispersion in the NR matrix was studied by SEM observation. As shown in Figure 10a, the agglomerate with a large size about $2000 \mathrm{~nm}$ is present in NR matrix. This indicates that the aggregation of lignin is unavoidable by co-precipitation method, although a part of lignin is dispersed in nanoscale due to the polydispersity of lignin. In contrast, it is clear to see that the lignin is uniformly dispersed in nanoscale in Figure 10a-10e. And the average particle size of LPCs calculated by Nano Measurer is about $90-100 \mathrm{~nm}$. The obvious differences in the two samples clarify that cationic PDADMAC polyelectrolytes can stabilize colloidal lignin particles and suppress aggregation when lignin is co-precipitated with NRL.

\subsection{Curing characteristics and crosslink density}

The curing characteristics of the pristine NR and its composites with lignin or LPCs, expressed in terms of the vulcanization times, $t_{\mathrm{S} 2}$ (scorch time) and $t_{90}$ (optimum cure time), as well as the maximum and minimum values of the torque, $S_{\max }$ and $S_{\min }$, respectively, and delta torque $\Delta S$ ( $\left.\Delta S=S_{\max }-S_{\min }\right)$, were deduced from curing curves. These parameters, along with the cure rate index, CRI expressed as CRI = $100 /\left(t_{90}-t_{\mathrm{S} 2}\right)$, were compiled as shown in Table 2 , as well as the crosslink density of composites.

It is obvious that incorporation of lignin or LPCs into NR matrix has distinctly different effects on the curing characteristics of composites. For NR/lignin composites, the scorch time gradually decreases with addition of lignin while the optimum cure time increases slightly. According to the gradually decreased CRI of NR/lignin compounds, we can infer that lignin hinders vulcanization. It can be attributed to the fact that phenolic hydroxyl of lignin not only acts as an activating agent to reduce scorch time, but also reacts with curing system to increase optimum cure time because of its acidity [13, 45], which results in a gradually decreased crosslink density of NR/lignin composites with the addition of lignin. For NR/LPCs compounds, both vulcanization times, $t_{\mathrm{S} 2}$ and $t_{90}$, are sharply reduced with addition of LPCs, showing accelerated vulcanization with respect to that of pure NR and NR/lignin compounds. These results are confirmed by CRI and the crosslink density data, which show a significant 


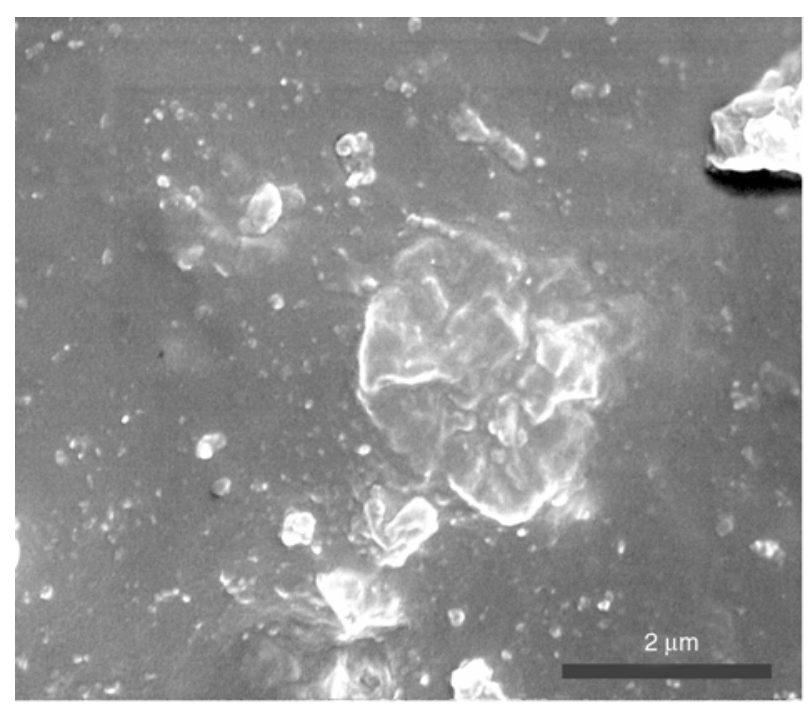

a)

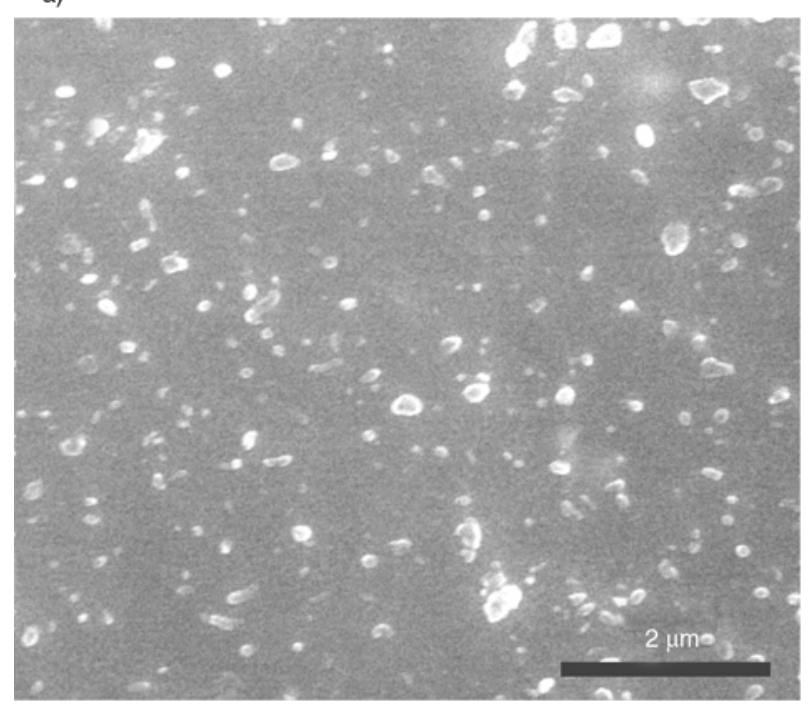

c)

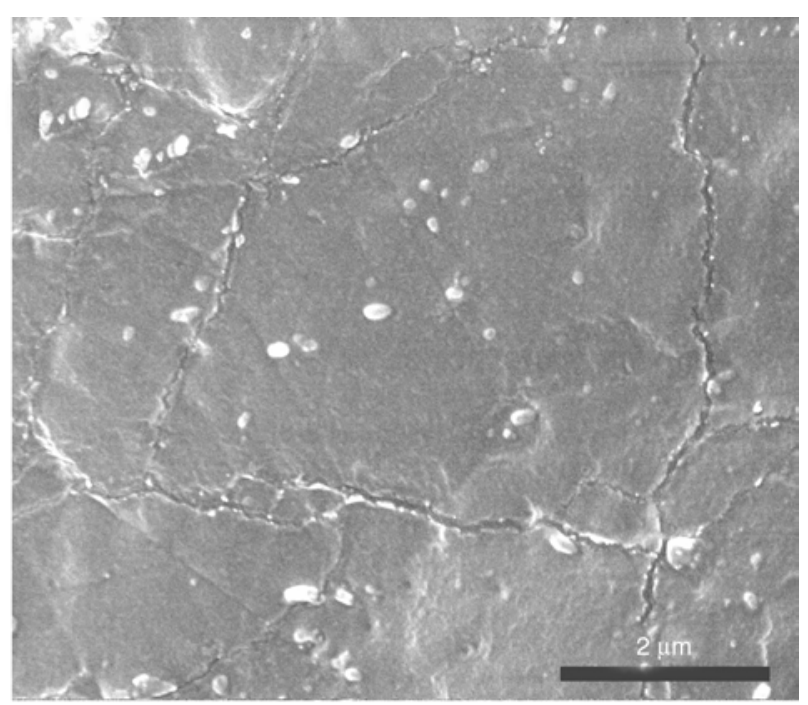

b)

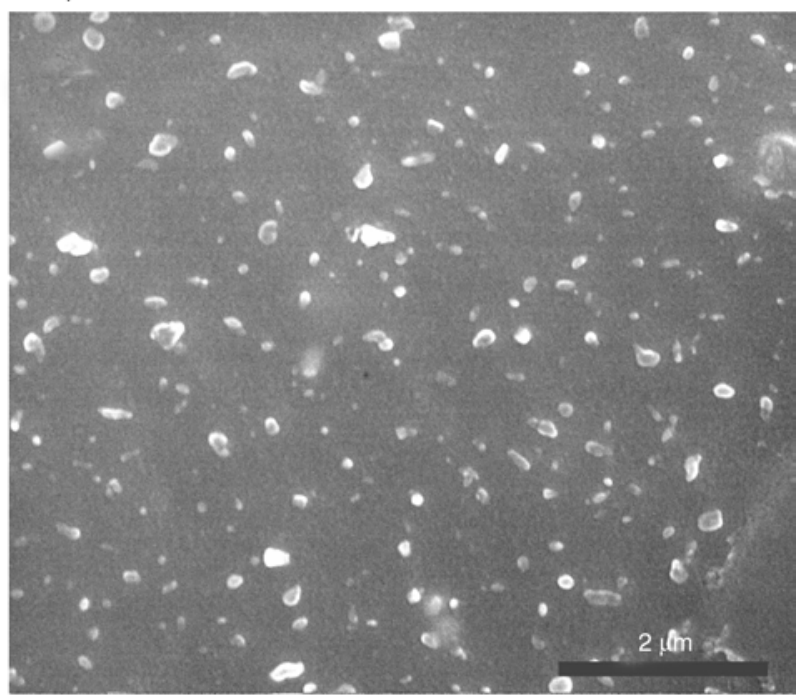

d)

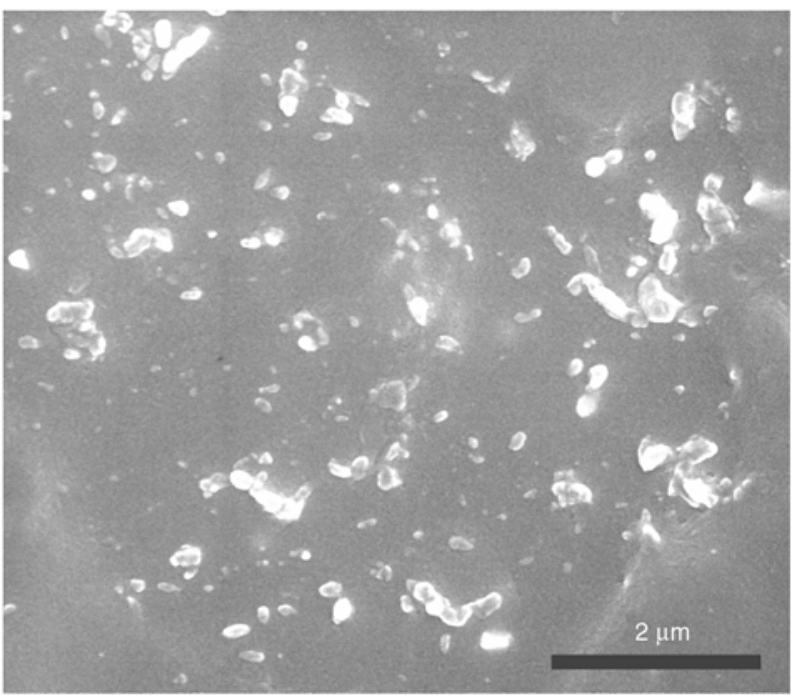

e)

Figure 10. SEM photos of NR/lignin composites and NR/LPCs nanocomposites. (a) L-7; (b) LPCs-1; (c) LPCs-3; (d) LPCs-5; (e) LPCs-7.

increase with increasing LPCs loading, mainly attributed to the accelerating effect provided by the
PDADMAC in LPCs [46, 47]. On the other hand, the minimum torque, maximum torque and delta torque 
Table 2. Curing characteristics and crosslink density of pure NR and its composites with lignin and LPCs

\begin{tabular}{|l|c|c|c|c|c|c|c|}
\hline Sample code & $\begin{array}{c}\mathbf{S}_{\mathbf{m i n}} \\
{[\mathbf{d N m}]}\end{array}$ & $\begin{array}{c}\mathbf{S}_{\mathbf{m a x}} \\
{[\mathbf{d N m}]}\end{array}$ & $\begin{array}{c}\mathbf{\Delta S} \\
{[\mathbf{d N m}]}\end{array}$ & $\begin{array}{c}\mathbf{t}_{\mathbf{S 2}} \\
{[\mathbf{m i n}]}\end{array}$ & $\begin{array}{c}\mathbf{t}_{\mathbf{9 0}} \\
{[\mathbf{m i n}]}\end{array}$ & $\begin{array}{c}\mathbf{C R I} \\
{\left[\mathbf{m i n}^{-\mathbf{1}}\right]}\end{array}$ & $\begin{array}{c}\text { Crosslink density } \\
{\left[\mathbf{1 0}^{-\mathbf{5}} \mathbf{m o l}_{\mathbf{c}} \mathbf{\mathbf { c m } ^ { 3 } ]}\right.}\end{array}$ \\
\hline L-0 & 0.09 & 10.58 & 10.49 & 19.55 & 31.45 & 8.40 & 6.65 \\
\hline L-1 & 0.17 & 11.90 & 11.73 & 18.56 & 32.40 & 7.23 & 6.57 \\
\hline L-3 & 0.15 & 11.70 & 11.55 & 18.16 & 32.54 & 6.95 & 5.47 \\
\hline L-5 & 0.16 & 11.31 & 11.15 & 17.34 & 32.15 & 6.75 & 5.25 \\
\hline L-7 & 0.19 & 12.42 & 12.23 & 17.20 & 33.15 & 6.27 & 5.13 \\
\hline LPCs-1 & 0.19 & 12.24 & 12.05 & 15.49 & 27.24 & 8.51 & 6.57 \\
\hline LPCs-3 & 0.25 & 12.68 & 12.43 & 10.17 & 18.52 & 11.98 & 6.63 \\
\hline LPCs-5 & 0.20 & 12.89 & 12.69 & 9.56 & 17.44 & 12.69 & 7.05 \\
\hline LPCs-7 & 0.35 & 14.50 & 14.15 & 8.20 & 15.33 & 14.03 & 7.21 \\
\hline
\end{tabular}

Table 3. Mechanical properties of pure NR and its composites with lignin and LPCs

\begin{tabular}{|l|c|c|c|c|c|}
\hline \multicolumn{1}{|c|}{ Sample code } & $\begin{array}{c}\text { Modulus at 300\% } \\
{[\mathbf{M P a}]}\end{array}$ & $\begin{array}{c}\text { Tensile strength } \\
{[\mathbf{M P a})}\end{array}$ & $\begin{array}{c}\text { Elongation at break } \\
{[\%]}\end{array}$ & $\begin{array}{c}\text { Tear strength } \\
{[\mathbf{k N} / \mathbf{m}]}\end{array}$ & $\begin{array}{c}\text { Hardness } \\
{[\text { Shore A] }}\end{array}$ \\
\hline L-0 & $2.00 \pm 0.03$ & $25.24 \pm 0.38$ & $654 \pm 13$ & $27.70 \pm 1.02$ & 38 \\
\hline L-1 & $2.14 \pm 0.16$ & $25.07 \pm 1.22$ & $679 \pm 16$ & $24.79 \pm 1.14$ & 36 \\
\hline L-3 & $1.96 \pm 0.00$ & $23.91 \pm 0.58$ & $701 \pm 13$ & $23.50 \pm 1.28$ & 37 \\
\hline L-5 & $2.13 \pm 0.03$ & $23.38 \pm 1.18$ & $675 \pm 13$ & $22.11 \pm 0.89$ & 38 \\
\hline L-7 & $2.17 \pm 0.07$ & $23.81 \pm 0.38$ & $693 \pm 4$ & $22.08 \pm 0.17$ & 39 \\
\hline LPCs-1 & $1.96 \pm 0.01$ & $25.69 \pm 1.30$ & $701 \pm 5$ & $27.35 \pm 0.43$ & 38 \\
\hline LPCs-3 & $2.25 \pm 0.09$ & $27.51 \pm 0.45$ & $715 \pm 14$ & $30.00 \pm 0.13$ & 40 \\
\hline LPCs-5 & $2.38 \pm 0.00$ & $27.91 \pm 0.46$ & $725 \pm 10$ & $28.12 \pm 1.05$ & 41 \\
\hline LPCs-7 & $2.95 \pm 0.10$ & $29.24 \pm 0.59$ & $658 \pm 20$ & $34.74 \pm 1.28$ & 44 \\
\hline
\end{tabular}

increase by addition of lignin or LPCs. It is of interest to point out that this increase is more evident for NR/LPCs composites, due to the higher crosslinking density of NR/LPCs composites.

\subsection{Mechanical property and dynamic mechanical property}

The mechanical properties of the pure NR and its composites with lignin or LPCs are listed in Table 3. Compared to pure NR, gradually decreased tensile strength and tear strength with addition of lignin can be observed in NR/lignin composites due to the bad interfacial compatibility between polar lignin and nonpolar NR, whereas a noticeable improvement in tensile strength, tear strength and the modulus at $300 \%$ is achieved with increasing LPCs loading in NR/LPCs composites. This distinction can be attributed to the two factors: lignin dispersion and affinity between rubber matrix and lignin. Lignin is well-dispersed in NR/LPCs composites and aggregates in NR/lignin composites, which has been disclosed by SEM. Moreover, LPCs has higher affinity toward NR matrix than lignin, due to the similarity between the chain backbones of PDADMAC on LPCs surface and natural rubber. Interestingly, the hardness of NR/lignin composites shows practically no variation, whereas that of NR/LPCs composites mildly increases with addition of LPCs. The increased hardness may be ascribed to the presence of PDADMAC.

The dynamic mechanical properties of NR/LPCs composites and NR/lignin composites were also performed. The variation of tangent delta $(\tan \delta)$ and storage modulus $\left(E^{\prime}\right)$ as a function of temperature as a comparison are reported in Figure 11. The $\tan \delta$ peak height is 1.58 and 1.64 respectively for NR/LPCs composites and NR/lignin composites. Furthermore, the glass transition temperature of $\mathrm{NR} / \mathrm{LPCs}$ composites is $-88^{\circ} \mathrm{C}$, which is $5^{\circ} \mathrm{C}$ higher than that of NR/lignin composites. These results suggest that there is a stronger interaction between LPCs and NR matrix. This interaction restricts the mobility of the elastomer segments, which significantly elevates the glass transition temperature. The $E^{\prime}$ of NR/LPCs composites shows a higher value than that of NR/lignin composites below the glass transition temperature, which demonstrates that the addition of LPCs into NR matrix results in an increase of stiffness. That further reflects the stronger confinement of LPCs on the rubber chains. 


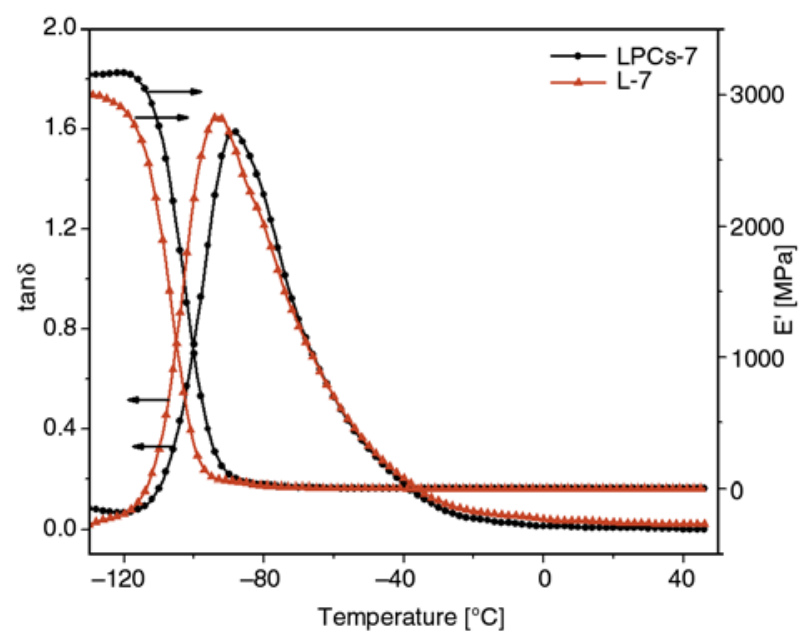

Figure 11. The curves of tangent delta and storage modulus versus the temperature for NR/lignin (L-7) and NR/LPCs (LPCs-7) composites

\subsection{Thermal and thermo-oxidative stability}

The thermal and thermo-oxidative stability of pure NR, NR/lignin and NR/LPCs composites can be assessed, respectively, from the investigation of thermal and thermo-oxidative decomposition. Figure 12a shows the DTG curves of pure NR, NR/ lignin and NR/LPCs composites in nitrogen. There is only one obvious thermal decomposition step of NR molecular chains, primarily initiated by thermal scissions of $\mathrm{C}-\mathrm{C}$ chain bonds accompanying a transfer of hydrogen at the site of scission.

The thermo-oxidative decomposition is obviously different from the thermal decomposition as shown in Figure 12b, which are DTG curves of pure NR, NR/lignin and NR/LPCs composites in air, respectively. There are three peaks in the DTG curve of pure NR, in contrast to NR/lignin and NR/LPCs composites. The first large decomposition peak $\left(358.8^{\circ} \mathrm{C}\right)$ is caused by the main oxidation of rubber. An addi-

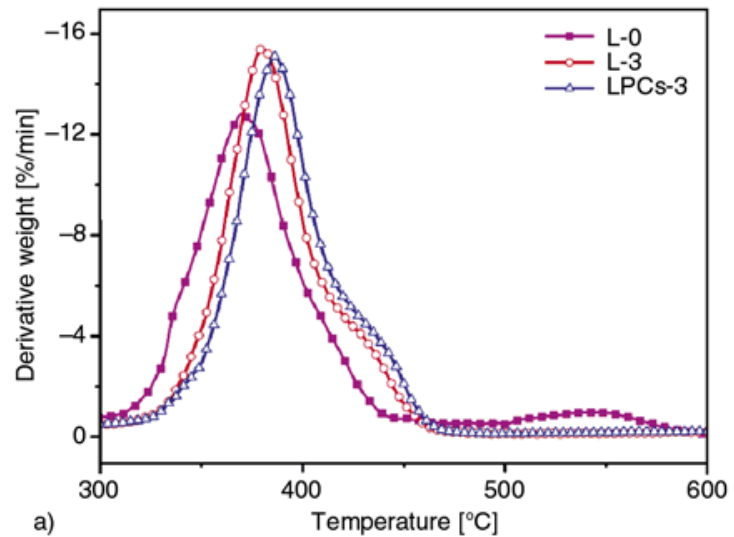

tional two small peaks probably originate from degradation of compounding ingredient $\left(440.4^{\circ} \mathrm{C}\right)$ and from the oxidation reaction of residual carbon. From the DTG curves of NR/lignin and NR/LPCs composites, it can be seen that there are no peaks at $440.4^{\circ} \mathrm{C}$, probably because of the formation of ligninsulfur intermediates originated during vulcanization of lignin-containing NR composites [22, 48].

Table 4 shows the thermal and thermo-oxidative degradation characteristics of pure NR, NR/lignin and NR/LPCs composites. For thermal decomposition, the $5,50 \%$ weight loss and peak decomposition temperature $\left(T_{\max }\right)$ of NR/LPCs composites shift to higher values, compared with pure NR and NR/lignin composites. The enhanced thermal stability can be contributed to the nanoscale dispersion of LPCs in NR matrix, which results in the LPCs and NR molecular chains strongly interacted through various effects such as the branching effect, nucleation effect, size effect and surface effect [49]. However, the change of those characteristic temperatures in thermo-oxidative decomposition is more complicated. Except the $T_{\max }$, other characteristic temperatures of NR/LPCs composites are inferior to that of NR/lignin composites. It is known that lignin's hindered phenolic hydroxyls can act as a

Table 4. Characteristic temperatures of thermal and thermooxidative decomposition for pure NR, NR/lignin and NR/LPCs composites

\begin{tabular}{|l|c|c|c|c|c|c|}
\hline \multirow{2}{*}{$\begin{array}{c}\text { Sample } \\
\text { code }\end{array}$} & \multicolumn{3}{|c|}{$\begin{array}{c}\text { Thermal } \\
\text { decomposition }\end{array}$} & \multicolumn{3}{c|}{$\begin{array}{c}\text { Thermo-oxidative } \\
\text { decomposition }\end{array}$} \\
\cline { 2 - 7 } & $\mathbf{T}_{\mathbf{5} \%}$ & $\mathbf{T}_{\mathbf{5 0}}$ & $\mathbf{T}_{\max }$ & $\mathbf{T}_{\mathbf{5} \%}$ & $\mathbf{T}_{\mathbf{5 0} \%}$ & $\mathbf{T}_{\mathbf{m a x}}$ \\
& {$\left[{ }^{\circ} \mathbf{C}\right]$} & {$\left[{ }^{\circ} \mathbf{C}\right]$} & {$\left[{ }^{\circ} \mathbf{C}\right]$} & {$\left[{ }^{\circ} \mathbf{C}\right]$} & {$\left[{ }^{\circ} \mathbf{C}\right]$} & {$\left[{ }^{\circ} \mathbf{C}\right]$} \\
\hline L-0 & 283.5 & 375.1 & 370.0 & 289.2 & 372.8 & 358.8 \\
\hline L-3 & 310.7 & 385.4 & 381.0 & 294.3 & 379.6 & 368.6 \\
\hline LPCs-3 & 312.0 & 390.7 & 386.5 & 288.6 & 373.9 & 372.5 \\
\hline
\end{tabular}

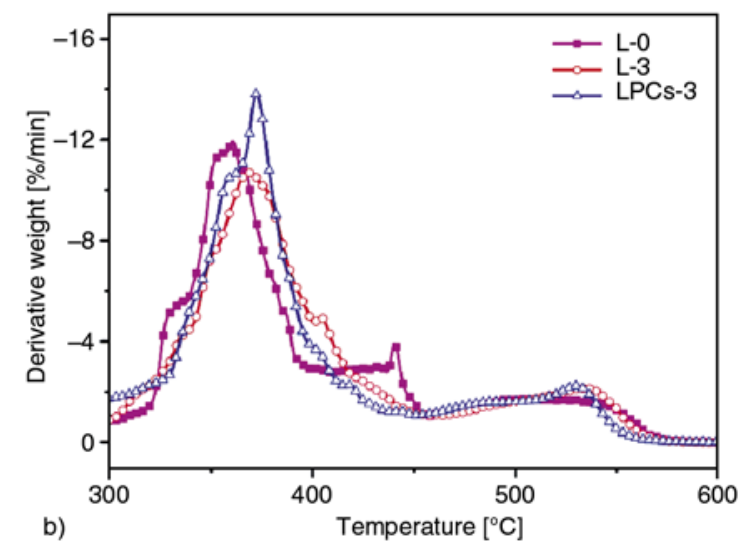

Figure 12. DTG curves of pure NR, NR/lignin (L-3) and NR/LPCs (LPCs-3) composites in nitrogen (a) and air (b) 
stabilizer of reactions induced by oxygen and its radical species [5]. The complicated results in thermo-oxidative decomposition may be contributed that the interactions between the phenolic hydroxyls of lignin and PDADMAC, such as cation- $\pi$ and $\pi-\pi$ interactions, weaken the ability of scavenging free radicals of phenolic hydroxyls in lignin.

\section{Conclusions}

Natural rubber/lignin nanocomposites were successfully prepared via co-precipitation of colloidal lignin-cationic polyelectrolyte complexes and rubber latex. PDADMAC was adsorbed onto lignin particles through ion-ion interactions, cation- $\pi$ and $\pi-\pi$ interactions which were confirmed by UV-vis and FTIR. As employing proper amount of PDADMAC, nanoscale LPCs were formed, laying a foundation for fabrication NR/LPCs nanocomposites. LPCs accelerated the vulcanization of NR/LPCs nanocomposites. Furthermore, LPCs were homogenously distributed in NR matrix, which resulted in improved mechanical properties, thermal and thermo-oxidative stability of NR/LPCs composites. In a word, this paper presents a promising strategy to utilize lignin for partial carbon black replacement.

\section{Acknowledgements}

The authors gratefully acknowledge National Natural Science Foundation of China (U1134005/L04) for financial supports.

\section{Referces}

[1] Vainio U., Maximova N., Hortling B., Laine J., Stenius P., Simola L. K., Gravitis J., Serimaa R.: Morphology of dry lignins and size and shape of dissolved kraft lignin particles by X-ray scattering. Langmuir, 20, 9736-9744 (2004).

DOI: $10.1021 / 1 \mathrm{a} 048407 \mathrm{v}$

[2] Gosselink R. J. A., Abächerli A., Semke H., Malherbe R., Käuper P., Nadif A., van Dam J. E. G.: Analytical protocols for characterisation of sulphur-free lignin. Industrial Crops and Products, 19, 271-281 (2004). DOI: $10.1016 /$ j.indcrop.2003.10.008

[3] De Paoli M-A., Furlan L. T.: Sugar cane bagasselignin as photo-stabilizer for butadiene rubber. Polymer Degradation and Stability, 11, 327-337 (1985). DOI: 10.1016/0141-3910(85)90036-9

[4] Boeriu C. G., Bravo D., Gosselink R. J. A., van Dam J. E. G.: Characterisation of structure-dependent functional properties of lignin with infrared spectroscopy. Industrial Crops and Products, 20, 205-218 (2004). DOI: $10.1016 /$ j.indcrop.2004.04.022
[5] Pouteau C., Dole P., Cathala B., Averous L., Boquillon N.: Antioxidant properties of lignin in polypropylene. Polymer Degradation and Stability, 81, 9-18 (2003). DOI: 10.1016/S0141-3910(03)00057-0

[6] Barclay L. R. C., Xi F., Norris J. Q.: Antioxidant properties of phenolic lignin model compounds. Journal of Wood Chemistry and Technology, 17, 73-90 (1997). DOI: 10.1080/02773819708003119

[7] Thielemans W., Wool R. P.: Lignin esters for use in unsaturated thermosets: Lignin modification and solubility modeling. Biomacromolecules, 6, 1895-1905 (2005).

DOI: $10.1021 / \mathrm{bm} 0500345$

[8] Li Y., Sarkanen S.: Alkylated kraft lignin-based thermoplastic blends with aliphatic polyesters. Macromolecules, 35, 9707-9715 (2002).

DOI: $10.1021 / \mathrm{ma} 021124 \mathrm{u}$

[9] Feldman D., Banu D., Campanelli J., Zhu H.: Blends of vinylic copolymer with plasticized lignin: Thermal and mechanical properties. Journal of Applied Polymer Science, 81, 861-874 (2001).

DOI: $10.1002 / a p p .1505$

[10] Hirose S., Kobashigawa K., Izuta Y., Hatakeyama H.: Thermal degradation of polyurethanes containing lignin studied by TG-FTIR. Polymer International, 47, 247256 (1998).

DOI: $10.1002 /(\mathrm{SICI}) 1097-0126(199811) 47: 3<247::$ AID-PI966>3.0.CO;2-F

[11] Setua D. K., Shukla M. K., Nigam V., Singh H., Mathur G. N.: Lignin reinforced rubber composites. Polymer Composites, 21, 988-995 (2000).

DOI: $10.1002 / p c .10252$

[12] Griffith T. R., MacGregor D. W.: Aids in vulcanization of lignin-natural rubber coprecipitates. Industrial and Engineering Chemistry, 45, 380-386 (1953). DOI: $10.1021 /$ ie50518a039

[13] Kumaran M. G., De S. K.: Utilization of lignins in rubber compounding. Journal of Applied Polymer Science, 22, 1885-1893 (1978). DOI: 10.1002/app.1978.070220711

[14] Benko D. A., Hahn B. R., Cohen M. P., Dirk, S. M., Cicotte K. N.: Functionalized lignin, rubber containing functionalized lignin and products containing such rubber composition. U.S. Patent 2010/0204368 A1, USA (2010).

[15] Boutsicaris S. P.: Lignin reinforced synthetic rubber. U.S. Patent 4477612, USA (1984).

[16] Davidson M. J. G., Wunder R. H.: Latex coagulation process using lignin compound. U.S. Patent 4025711, USA (1977).

[17] Doughty J. B.: Method of dry-milling carboxylic elastomers and alkali lignins. U.S. Patent 3325427, USA (1967).

[18] Doughty J. B., Charlestion S. C.: Lignin reinforced rubber and method of preparation thereof. U.S. Patent 3247135, USA (1966). 
[19] Sirianni A. F.: Puddington I. E.: Reinforcing polymers with laundered amorphous lignin. U.S. Patent 3817974, USA (1976).

[20] Keilen J. J., Pollak A.: Lignin for reinforcing rubber. Industrial and Engineering Chemistry, 39, 480-483 (1947).

DOI: $10.1021 /$ ie 50448a013

[21] Botros S. H., Eid M. A. M., Nageeb Z. A.: Thermal stability and dielectric relaxation of natural rubber/ soda lignin and natural rubber/thiolignin composites. Journal of Applied Polymer Science, 99, 2504-2511 (2006).

DOI: 10.1002/app.22865

[22] Košíková B., Gregorová A.: Sulfur-free lignin as reinforcing component of styrene-butadiene rubber. Journal of Applied Polymer Science, 97, 924-929 (2005). DOI: 10.1002 /app. 21448

[23] Maximova N.: Adsorption of lignin and lignin/cationic polymer complexes on cellulose fibres and their effect on sheet properties. $\mathrm{PhD}$ thesis, Helsinki University of Technology (2004).

[24] Lappan R. E., Pelton R., McLennan I., Patry J., Hrymak A. N.: Kraft lignin-poly(DADMAC) precipitate formation. Industrial and Engineering Chemistry Research, 36, 1171-1175 (1997).

DOI: $10.1021 /$ ie $960460 \mathrm{u}$

[25] Li P., Pelton R.: Wood pulp washing 1. Complex formation between kraft lignin and cationic polymers. Colloids and Surfaces, 64, 217-222 (1992).

DOI: 10.1016/0166-6622(92)80102-8

[26] Vanerek A., van de Ven T. G. M.: Coacervate complex formation between cationic polyacrylamide and anionic sulfonated kraft lignin. Colloids and Surfaces A: Physicochemical and Engineering Aspects, 273, 5562 (2006).

DOI: 10.1016/j.colsurfa.2005.08.005

[27] Gregorová A., Košíková B., Moravčík R.: Stabilization effect of lignin in natural rubber. Polymer Degradation and Stability, 91, 229-233 (2006). DOI: 10.1016/j.polymdegradstab.2005.05.009

[28] Lindströmn T.: The colloidal behaviour of kraft lignin. Colloid and Polymer Science, 257, 277-285 (1979). DOI: 10.1007/BF01382370

[29] Norgren M., Edlund H., Wågberg L., Lindström B., Annergren G.: Aggregation of kraft lignin derivatives under conditions relevant to the process, Part I: Phase behaviour. Colloids and Surfaces A: Physicochemical and Engineering Aspects, 194, 85-96 (2001).

DOI: 10.1016/S0927-7757(01)00753-1

[30] Lindström T., Westman L.: The colloidal behaviour of kraft lignin. Colloid and Polymer Science, 260, 594598 (1982).

DOI: $10.1007 / \mathrm{BF} 01422591$

[31] Dong D., Fricke A. L., Moudgil B. M., Johnson H.: Electrokinetic study of kraft lignin. Tappi Journal, 79, 191-197 (1996).
[32] Lindström T.: The colloidal behaviour of kraft lignin. Colloid and Polymer Science, 258, 168-173 (1980). DOI: $10.1007 / \mathrm{BF} 01498276$

[33] Chernoberezhskii Y. M., Atanesyan A. A., Dyagileva A. B., Lorentsson A. V., Leshchenko T. V.: Effect of the concentration of sulfate lignin on the aggregation stability of its aqueous dispersions. Colloid Journal, 64, 637-639 (2002).

DOI: 10.1023/A:1020678430092

[34] Norgren M., Edlund H.: Stabilisation of kraft lignin solutions by surfactant additions. Colloids and Surfaces A: Physicochemical and Engineering Aspects, 194, 239-248 (2001).

DOI: $10.1016 / \mathrm{S} 0927-7757(01) 00806-8$

[35] Norgren M., Lindström B.: Dissociation of phenolic groups in kraft lignin at elevated temperatures. Holzforschung, 54, 519-527 (2000).

DOI: $10.1515 /$ HF.2000.088

[36] Micic M., Benitez I., Ruano M., Mavers M., Jeremic M., Radotic K., Moy V., Leblanc R. M.: Probing the lignin nanomechanical properties and lignin-lignin interactions using the atomic force microscopy. Chemical Physics Letters, 347, 41-45 (2001). DOI: 10.1016/S0009-2614(01)01022-3

[37] Mičič M., Jeremič M., Radotič K., Mavers M., Leblanc R. M.: Visualization of artificial lignin supramolecular structures. Scanning, 22, 288-294 (2000). DOI: $10.1002 /$ sca.4950220503

[38] Sarkanen S., Teller D. C., Stevens C. R., McCarthy J. L.: Lignin. 20. Associative interactions between kraft lignin components. Macromolecules, 17, 2588-2597 (1984). DOI: $10.1021 / \mathrm{ma} 00142 \mathrm{a} 022$

[39] Sarkanen S., Teller D. C., Abramowski E., McCarthy J. L.: Lignin. 19. Kraft lignin component conformation and associated complex configuration in aqueous alkaline solution. Macromolecules, 15, 1098-1104 (1982). DOI: $10.1021 / \mathrm{ma} 00232 \mathrm{a} 027$

[40] Pogodina N. V., Tsvetkov N. V.: Structure and dynamics of the polyelectrolyte complex formation. Macromolecules, 30, 4897-4904 (1997).

DOI: $10.1021 / \mathrm{ma9617983}$

[41] Ouyang X., Deng Y., Qian Y., Zhang P., Qiu X.: Adsorption characteristics of lignosulfonates in saltfree and salt-added aqueous solutions. Biomacromolecules, 12, 3313-3320 (2011).

DOI: $10.1021 / \mathrm{bm} 200808 \mathrm{p}$

[42] Deng Y., Zhang W., Wu Y., Yu H., Qiu X.: Effect of molecular weight on the adsorption characteristics of lignosulfonates. The Journal of Physical Chemistry B, 115, 14866-14873 (2011).

DOI: $10.1021 / j p 208312 \mathrm{a}$

[43] Pillai K. V., Renneckar S.: Cation- $\pi$ interactions as a mechanism in technical lignin adsorption to cationic surfaces. Biomacromolecules, 10, 798-804 (2009). DOI: $10.1021 / \mathrm{bm} 801284 \mathrm{y}$ 
[44] Yang D-Q., Rochette J-F., Sacher E.: Spectroscopic evidence for $\pi-\pi$ interaction between poly(diallyl dimethylammonium) chloride and multiwalled carbon nanotubes. The Journal of Physical Chemistry B, 109, 4481-4484 (2005).

DOI: $10.1021 / j p 044511+$

[45] Nando G. B., De S. K.: Effect of lignin on the network structure and properties of natural rubber mixes vulcanized by conventional, semiefficient and efficient vulcanization systems. Journal of Applied Polymer Science, 25, 1249-1252 (1980).

DOI: 10.1002/app.1980.070250625

[46] Verezhnikov V. N., Nikulin S. S., Poyarkova T. N., Misin V. M.: Separation of styrene-butadiene rubbers from latexes using dimethyldiallylammonium chloride- $\mathrm{SO}_{2}$ copolymer. Russian Journal of Applied Chemistry, 74, 1225-1229 (2001).

DOI: $10.1023 / \mathrm{A}: 1013004025985$
[47] Verezhnikov V. N., Nikulin S. S., Krutikov M. Y., Poyarkova T. N.: Kinetics of latex flocculation by cationic polyelectrolyte from data of nephelometry (in Russian). Colloid Journal of the Russian Academy of Sciences, 61, 37-40 (1999).

[48] Košíková B., Gregorová A., Osvald A., Krajčovičová J.: Role of lignin filler in stabilization of natural rubber-based composites. Journal of Applied Polymer Science, 103, 1226-1231 (2007).

DOI: $10.1002 / a p p .24530$

[49] Peng Z., Kong L. X., Li S-D., Chen Y., Huang M. F.: Self-assembled natural rubber/silica nanocomposites: Its preparation and characterization. Composites Science and Technology, 67, 3130-3139 (2007). DOI: 10.1016/j.compscitech.2007.04.016 